\title{
Modulation of cardiac contractility by muscle metaboreflex following efforts of different intensities in humans
}

\author{
Antonio Crisafulli, ${ }^{1,2,3}$ Enrico Salis, ${ }^{1}$ Gianluigi Pittau, ${ }^{1}$ Luigi Lorrai, ${ }^{1}$ \\ Filippo Tocco, ${ }^{1}$ Franco Melis, ${ }^{1}$ Pasquale Pagliaro, ${ }^{2}$ and Alberto Concu ${ }^{1}$ \\ ${ }^{1}$ Department of Science Applied to Biological Systems, University of Cagliari, Cagliari; \\ ${ }^{2}$ Department of Biological Science, University of Torino, Torino; and ${ }^{3}$ Department of \\ Biomedical Sciences, Human Physiology Division, University of Sassari, Sassari, Italy
}

Submitted 1 March 2006; accepted in final form 7 June 2006

Crisafulli, Antonio, Enrico Salis, Gianluigi Pittau, Luigi Lorrai, Filippo Tocco, Franco Melis, Pasquale Pagliaro, and Alberto Concu. Modulation of cardiac contractility by muscle metaboreflex following efforts of different intensities in humans. Am J Physiol Heart Circ Physiol 291: H3035-H3042, 2006. First published June 16, 2006; doi:10.1152/ajpheart.00221.2006.—Accumulation of metabolic end products within skeletal muscle stimulates sensory nerves, thus evoking a pressor response termed "metaboreflex." The aim of this study was to evaluate changes in hemodynamics occurring during metaboreflex activation obtained by postexercise muscle ischemia (PEMI) after two different exercise intensities. In twelve healthy subjects, the metaboreflex was studied with the PEMI method at the start of recovery from one leg-dynamic knee extension performed at intensities of 30\% (PEMI 30\%) and 70\% (PEMI 70\%) of the maximum workload achieved in a preliminary test. Control exercise recovery tests at the same intensities were also conducted. Central hemodynamics were evaluated by means of impedance cardiography. The main findings were that 1 ) during metaboreflex, exercise conducted against the higher workload caused a more pronounced blood pressure increase than the strain conducted against the lower workload; and 2) during PEMI 70\%, this blood pressure response was mainly achieved through enhancement of myocardial contractility that increased stroke volume and, in turn, cardiac output, whereas during PEMI $30 \%$, the blood pressure response was reached predominantly by means of vasoconstriction. Thus a substantial enhancement of myocardial contractility was reached only in the PEMI $70 \%$ test. These results suggest that hemodynamic regulation during metaboreflex engagement caused by PEMI in humans is dependent on the intensity of the previous effort. Moreover, the cardiovascular response during metaboreflex is not merely achieved by vasoconstriction alone, but it appears that there is a complex interplay between peripheral vasoconstriction and heart contractility recruitment.

stroke volume; cardiac output; blood pressure; exercise; impedance cardiography

DURING EXERCISE, neural signals of both central and peripheral origin provide information to the cardiovascular control areas responsible for hemodynamic regulation. Such information aims at adjusting the cardiovascular system to muscle requirements. In particular, the activation of regions of the brain responsible for motor unit recruitment also activates the cardiovascular control areas, thus establishing a basal level of sympathetic and parasympathetic efferent activity to the cardiovascular apparatus closely linked to the intensity of the effort $(39,40)$. This basic pattern of autonomic activity, com-

Address for reprint requests and other correspondence: A. Crisafulli, Dept. of Science Applied to Biological Systems, Section of Human Physiology, School of Sport Medicine, Univ. of Cagliari, Via Porcell 4, 09124 Cagliari, Italy (e-mail: crisafulli@tiscali.it). monly known as "central command," is in turn modulated by peripheral signals originating from receptors within muscle that reflexively activate the cardiovascular control centers $(25$, 29, 32, 39).

The current thinking is that when $\mathrm{O}_{2}$ delivery does not suffice to meet the requirements of contracting muscles, metabolic end products accumulate and activate muscle metaboreceptors (free endings of group III and IV afferents), which raise arterial blood pressure through a reflex of nervous origin commonly called "metaboreflex." This pressure effect is thought to be mostly mediated by reflex vasoconstriction, i.e., by a systemic vascular resistance (SVR) increase that elevates blood pressure to restore blood flow to the hypoperfused muscle $(26,28,34)$. Actually, the stimulation of group III and/or IV afferents appears to be essential for the normal hemodynamic response to exercise, because its absence abolishes the normal increase in blood pressure commonly observed during effort (39).

However, whereas the effect of metaboreflex on SVR has been well established, less is known about its action on central hemodynamics, i.e., myocardial contractility, cardiac preload, stroke volume $(\mathrm{SV})$, and cardiac output $(\mathrm{CO})$. A number of studies suggest that metaborflex activation can also affect central hemodynamics. For example, it has been found in dogs that the muscle metaboreflex is capable of increasing ventricular performance $(27,33)$. Similarly, it has been reported in humans that myocardial contractility and SV can be improved during metaboreflex activation caused by postexercise muscle ischemia $(5,6,9)$. Moreover, it has been proposed that the muscle metaboreflex is capable of enhancing filling pressure through splanchnic and general venoconstriction that propels blood volume toward the central circulation (1a, 35). Hence, from the aforementioned results, it appears that the metaboreflex may modulate peripheral as well as central hemodynamics.

It has been suggested that the intensity of the exercise performed before metaboreflex stimulation may determine the mechanism on which blood pressure response relies $(13,14)$. In particular, some studies $(1,34,41)$ conducted on animals reported that cardiovascular response to metaboreflex relies mainly on $\mathrm{CO}$ during mild exercise, whereas peripheral vasoconstriction becomes more important as exercise intensity rises. It is believed that if the contractile potentialities of the heart are not fully exploited during mild exercise (i.e., the cardiac reserve can be still used), then the subsequent

The costs of publication of this article were defrayed in part by the payment of page charges. The article must therefore be hereby marked "advertisement" in accordance with 18 U.S.C. Section 1734 solely to indicate this fact. 
metaboreflex-induced pressure response relies mainly on $\mathrm{CO}$, whereas if the cardiac reserve is utilized during and/or after strenuous efforts, then the reflex mainly relies on peripheral vasoconstriction. Alternatively, inasmuch as it was demonstrated that strains performed at a heavier workload may cause a more powerful metaboreflex engagement (i.e., blood pressure increment) than strains conducted at lighter intensities because of differences in the concentration of muscle metabolites (7, 22 ), it can be argued that exercises conducted at higher intensities may also recruit a more powerful cardiac response in term of contractility recruitment. It is thus likely that the hemodynamic response to metaboreflex is the result of the combination between the degree of metaboreceptors stimulation, which depends on metabolite accumulation, and the possibility of increasing $\mathrm{CO}$, which mainly depends on the availability of a contractility reserve. Importantly, both the degree of metabolite accumulation and the availability of a cardiac reserve depend on the intensity of the effort. All these facts seem to indicate that the cardiovascular apparatus operates with remarkable plasticity to regulate the cardiovascular response to the metaboreflex, which may be the result of a complex interplay between the sympathetic stimulation due to metabolite accumulation and the cardiovascular response, which is probably not merely constituted by peripheral vasoconstriction.

However, to the best of our knowledge, there are no human studies comparing central and peripheral hemodynamic responses during metaboreflex engagement after efforts of different intensities in the same individuals in two different exercise sessions. Therefore, we determined to study whether or not metaboreflex stimulation after exercises performed at different intensities leads to a different response in terms of vascular resistance, myocardial contractility, $\mathrm{SV}$, and $\mathrm{CO}$.

\section{METHODS}

Study population. Twelve healthy men between the ages of 23 and $37 \mathrm{yr}$ (mean $\pm \mathrm{SE}, 28.3 \pm 1.3 \mathrm{yr}$ ), whose height and mass were $175.8 \pm 1.4 \mathrm{~cm}$ and $72.9 \pm 1.9 \mathrm{~kg}$, respectively, agreed to participate in this study. None had any history of cardiac or respiratory disease or were taking any medication at the time of the experiment. All subjects were normotensive, and none showed any abnormalities on physical examination. The study was performed according to the Declaration of Helsinki and was approved by a local ethics committee. All subjects gave written informed consent.

Experimental design. Before entering the study, each subject underwent a preliminary one leg-dynamic knee extension test on a computer-controlled multifunctional dynamometer device (REV 9000 , Technogym, Forlì, Italy) to assess the maximum workload achievable $\left(\mathrm{W}_{\max }\right)$. Subjects were seated on the chair of the dynamometer, placed with the upper part of the leg horizontal and the dominant knee flexed at $90^{\circ}$. The lower third of the exercising leg was attached to the distal end of the lever arm of the dynamometer. The nonexercising leg was maintained flexed at $90^{\circ}$ at the knee. Dynamic exercise was performed by the dominant leg at a rate of 30 contractions/min against adjustable workloads, with one contraction causing the exercising leg to move from $90^{\circ}$ to $150^{\circ}$ knee extension. The workload was increased by steps of $5 \mathrm{~W} / \mathrm{min}$, starting from $5 \mathrm{~W}$, up to $\mathrm{W}_{\max }$, which was considered the point at which the subject was no longer able to maintain the contracting rate.

Subjects then underwent the following study protocol assigned in random order on the same dynamometer previously described. First, postexercise muscle ischemia session (PEMI) after exercise at $30 \%$ of $\mathrm{W}_{\max }($ PEMI $30 \%$ ): after $3 \mathrm{~min}$ of rest, subjects performed $5 \mathrm{~min}$ of exercise consisting of dynamic knee extension against a workload corresponding to $30 \%$ of $\mathrm{W}_{\max }$ at a contracting rate of 30 contractions/min. At the end of the exercise, we induced PEMI by rapidly (in $<3 \mathrm{~s}$ ) inflating a pneumatic cuff, previously placed as high as possible on the thigh, to $50 \mathrm{mmHg}$ above peak exercise arm systolic pressure. The cuff was kept inflated for $3 \mathrm{~min}$. Three minutes of recovery were further allowed after the cuff was deflated, for a total of 6 min of recovery. Second, a control exercise recovery session (CER) after exercise at $30 \%$ of $\mathrm{W}_{\max }(\mathrm{CER} 30 \%$ ): the same rest-exercise protocol used for PEMI $30 \%$ test was conducted, followed by a control exercise recovery of 6 min without tourniquet inflation. Third, PEMI after exercise at $70 \%$ of $\mathrm{W}_{\max }$ (PEMI 70\%): the same rest-exerciserecovery protocol used for PEMI $30 \%$ test was employed, but subjects exercised against a workload of $70 \%$ of $\mathrm{W}_{\max }$. Fourth, CER at $70 \%$ of $\mathrm{W}_{\max }$ (CER 70\%): subjects underwent the same rest-exercise-recovery protocol used for CER 30\% test, but they exercised against a workload of $70 \%$ of $\mathrm{W}_{\text {max }}$. Fifth, control-rest test (CR test): on this day each subject reported to the laboratory and sat quietly on the chair of the dynamometer for $14 \mathrm{~min}$ to obtain reference control rest values in the same environmental conditions as the previous protocol periods. This session was applied to obtain a reference control rest with which to compare data from the other tests. All experimental sessions were carried out in a temperature-controlled, air-conditioned room and at intervals of at least 3 days.

Hemodynamic and ventilatory data. Hemodynamic and ventilatory data were recorded throughout all phases of the protocol. Hemodynamic parameters were measured by means of impedance cardiography (NCCOM 3, BoMed, Irvine, CA), which allows continuous noninvasive cardiodynamic measuring during exercise and recovery $(3,8,10,11,24)$. The impedance method assumes that when an electrical current circulates through the thorax, the pulsatile aortic blood flow induces a proportional variation in electrical conductivity; hence, changes in thoracic electrical impedance during systole are representative of SV (4). The device was connected to the subject by arranging eight spot electrodes: two pairs were thoracic and cervical injecting electrodes, whereas two other pairs were sensing electrodes placed above the cervical and below the thoracic pairs. By means of a digital chart recorder (ADInstruments, PowerLab 8sp, Castle Hill, Australia), we stored NCCOM 3-derived analog traces of electrocardiogram, thorax impedance $\left(Z_{0}\right)$, and $Z_{0}$ first derivative $(\mathrm{d} Z / \mathrm{d} t)$. Afterward, stored impedance traces were cleaned from signals affected by movement and respiratory artifacts and analyzed taking particular care to calculate hemodynamic variables only from traces not affected by impedance artifacts. We previously utilized this signal processing procedure, which, though time-consuming, allows the obtaining of reliable and reproducible hemodynamic data estimation during exercise and recovery $(8,10,11)$.

SV was assessed by using the Sramek-Bernstein equation (4):

$$
\mathrm{SV}=\left(\mathrm{VEPT} \cdot Z_{0}^{-1}\right) \cdot \mathrm{d} Z / \mathrm{d} t_{\max } \cdot \mathrm{VET}
$$

where VEPT was the volume of electrical participating tissue and was derived using a nomogram from sex, height, and weight of the subject; $Z_{0}$ was the thorax impedance measured at the end of cardiac diastole; $\mathrm{d} Z / \mathrm{d} t_{\max }$ was the maximal $Z_{0}$ first derivative during cardiac systole; and VET was the left ventricular ejection time, measured as the interval between the beginning and the minimum of the deflection in $\mathrm{d} Z / \mathrm{d} t$ trace during systole (8). Heart rate (HR) was calculated as the reciprocal of the electrocardiogram R-R interval, and $\mathrm{CO}$ was obtained by multiplying SV and HR. Also measured was the preejection period (PEP), identified as the time interval between the electrocardiogram $\mathrm{Q}$ wave and the beginning of the $\mathrm{d} Z / \mathrm{d} t$ deflection during systole (8). By calculating the PEP-to-VET (PEP/VET) ratio, we obtained an index inversely related to myocardial contractility $(8-10)$. Subjects were also connected to a noninvasive automated sphygmomanometer (NIBP 7000, Colin Medical Instrument, San Antonio, TX) that provided beat-to-beat values of systolic (SBP) and diastolic (DBP) blood pressure by means of a tonometer placed around the 
wrist, at the height of the radial artery. This device has been shown to provide continuous beat-to-beat pressure monitoring with high accuracy (21). We then used the formula proposed by Moran and coworkers (20) to calculate mean blood pressure (MBP). This formula allows calculation of MBP, taking into account changes in the diastolic and systolic periods caused by exercise tachycardia. The fraction of systole (FS) from the heart cycle was assessed, and MBP was calculated from DBP and the pulse pressure (PP) adjusted for FS as follows: DBP + FS $\cdot$ PP. SVR was derived by multiplying the MBPto- $\mathrm{CO}$ ratio by 80 , where 80 is a conversion factor to change units to standard resistance units.

Pulmonary ventilation $\left(\dot{\mathrm{V}}_{\mathrm{E}}\right)$, whole body oxygen consumption $\left(\dot{\mathrm{V}}_{2}\right)$, and carbon dioxide production $\left(\dot{\mathrm{V}}_{\mathrm{CO}_{2}}\right)$ were measured using a breath-by-breath mass spectrometry system (MedGraphics Breeze, St. Paul, MN), calibrated immediately before each exercise test.

To obtain an index of metabolite production during exercise, we also assessed the excess $\mathrm{CO}_{2}$ production $\left(\mathrm{CO}_{2}\right.$ excess $)$ as follows:

$$
\mathrm{Co}_{2 \mathrm{excess}}=\left(\dot{\mathrm{V}} \mathrm{CO}_{2}-\mathrm{RER}_{\mathrm{rest}} \cdot \dot{\mathrm{V}}_{2}\right) \text {, }
$$

where $\mathrm{RER}_{\text {rest }}$ is the respiratory exchange ratio at rest. $\mathrm{CO}_{2 \text { excess }}$ represents an index of lactic acid and $\mathrm{H}^{+}$accumulation because, at tissue $\mathrm{pH}$, lactic acid dissociates and produces $\mathrm{H}^{+}$, which is buffered by ${ }^{-} \mathrm{HCO}_{3}$ and other cell buffers. The amount being buffered by ${ }^{-} \mathrm{HCO}_{3}$ leads to $\mathrm{H}_{2} \mathrm{CO}_{3}$ production and then to $\mathrm{H}_{2} \mathrm{O}$ and $\mathrm{CO}_{2}$ (2). In this way, $\mathrm{CO}_{2}$ excess is produced and is superimposed on the $\mathrm{CO}_{2}$ normally deriving from aerobic metabolism. In fact, $\mathrm{CO}_{2 \text { excess }}$ was found to correlate well with the rate of lactate accumulation in the blood during exercise $(12,31)$.

Data analysis. Hemodynamic and ventilatory data at the 5th min of exercise and throughout recovery are shown. Data were averaged for $1 \mathrm{~min}$. Responses are reported as mean \pm SE percent changes from corresponding rest values. We considered rest values as the last of the 3 min preceding the exercise runs. Because the rest values of $\mathrm{CO}_{2 \text { excess }}$ are clearly equal to 0 , for this index we showed absolute values instead of percent changes from rest. Comparisons were performed using the two-way ANOVA for repeated measures (factors: condition and time) followed by Bonferroni post hoc test when appropriate by utilizing commercially available software (SigmaStat 2.03). Statistical significance was set at a $P$ value of $<0.05$ in all cases.

\section{RESULTS}

All subjects completed the protocol, and none of them complained of significant pain or discomfort during the periods of circulatory occlusion. No significant differences in rest variables before the four tests were observed (Table 1).
Figures 1-3 show cardiovascular and ventilatory responses during each protocol period.

As expected, HR (Fig. 1A) increased during all exercise sessions compared with that in the CR condition. Moreover, during the 5 th min of exercise, HR was higher at $70 \%$ than at $30 \%$ of $\mathrm{W}_{\max }$ tests. During the periods of recovery from efforts, HR rapidly decreased toward rest level, and there were no differences in CER 70\%, PEMI 30\%, and CER 30\% tests. However, during the 1 st min of recovery of PEMI $70 \%$, HR was still higher than the $\mathrm{CR}$ test and also higher with respect to the two tests performed at $30 \%$ of $\mathrm{W}_{\max }$.

SV (Fig. $1 B$ ) increased significantly during the two strains conducted at $70 \%$ of $\mathrm{W}_{\max }$ (PEMI or CER) but not during the two tests conducted at $30 \%$ of $\mathrm{W}_{\max }$. Importantly, during the recovery of CER $70 \%, \mathrm{SV}$ returned gradually to the CR test level, whereas during the 3 min of circulatory occlusion of the PEMI 70\% test, SV further increased. Therefore, throughout artery occlusion, SV during the PEMI $70 \%$ session was significantly higher than during the PEMI 30\% and CER 30\% tests. In the PEMI 70\% session, SV then promptly decreased once the circulatory occlusion was removed.

Cardiac output (Fig. 1C) rose during the 5 th min of effort in all exercise bouts compared with that in the CR test, and, as expected, this increment was more pronounced during efforts performed against $70 \%$ instead of $30 \%$ of $\mathrm{W}_{\text {max }}$. During the recovery periods of the PEMI 30\% and CER 30\% tests, CO gradually returned to the CR level, and there was no difference in the time course between these two settings. $\mathrm{CO}$ also decreased during recovery of the CER $70 \%$ test, showing values that were not different compared with those in the PEMI 30\% and CER 30\% tests, although it later returned to the CR level, i.e., at the 2 nd min of recovery. On the contrary, during the PEMI $70 \%$ test, CO recovery was slower than during the other protocol periods. Thus CO in this session resulted higher than in the PEMI 30\%, CER 30\%, and CR tests until the 3rd min of recovery; $\mathrm{CO}$ also resulted higher than in the CER $70 \%$ test to the limited extent of the 1 st min of recovery.

As concerns PEP/VET ratio (inversely related to contractility), it dropped (i.e., contractility increased) with respect to $\mathrm{CR}$ during the 5th min of exercise in the PEMI $70 \%$ and CER 70\% tests, whereas it was unchanged during the PEMI $30 \%$ and CER 30\% tests (Fig. 1D). During recovery in the CER 70\%

Table 1. Variables during rest preceding tests

\begin{tabular}{|c|c|c|c|c|c|c|}
\hline $\mathrm{SV}, \mathrm{ml}$ & $69.6 \pm 5.4$ & $71.8 \pm 3.6$ & $73.3 \pm 4.6$ & $73.6 \pm 3.7$ & $69.7 \pm 3.6$ & NS \\
\hline $\mathrm{SBP}, \mathrm{mmHg}$ & $109.5 \pm 5.3$ & $115.4 \pm 3.7$ & $111.7 \pm 4.8$ & $108.9 \pm 4.6$ & $110.8 \pm 4.1$ & NS \\
\hline $\mathrm{DBP}, \mathrm{mmHg}$ & $76.6 \pm 3.3$ & $73.3 \pm 1.7$ & $73.4 \pm 1.8$ & $75.1 \pm 2$ & $76.6 \pm 3.3$ & NS \\
\hline $\mathrm{MBP}, \mathrm{mmHg}$ & $87.7 \pm 1.5$ & $87.8 \pm 2.1$ & $85.8 \pm 2.2$ & $85.9 \pm 2.6$ & $88 \pm 1.7$ & NS \\
\hline $\mathrm{SVR}, \mathrm{dyn} \cdot \mathrm{s} \cdot \mathrm{cm}^{-5}$ & $1,428 \pm 72$ & $1,325 \pm 52$ & $1,327 \pm 99$ & $1,346 \pm 94$ & $1,450 \pm 76$ & NS \\
\hline$\dot{\mathrm{V}}_{\mathrm{CO}_{2}}, 1 / \mathrm{min}$ & $0.33 \pm 0.02$ & $0.34 \pm 0.03$ & $0.32 \pm 0.02$ & $0.34 \pm 0.01$ & $0.30 \pm 0.03$ & NS \\
\hline$\dot{\mathrm{V}}_{\mathrm{E}}, 1 / \mathrm{min}$ & $12.3 \pm 0.5$ & $13.8 \pm 0.4$ & $13.5 \pm 0.7$ & $13.4 \pm 0.5$ & $12.8 \pm 0.6$ & NS \\
\hline
\end{tabular}

Values are means \pm SE. PEMI $30 \%$ and PEMI $70 \%$, postexercise muscle ischemia at 30\% and 70\% intensities, respectively; CER 30\% and CER 70\%, control exercise recovery at $30 \%$ and $70 \%$ intensities, respectively; CR, control rest; HR, heart rate; SV, stroke volume; CO, cardiac output; SBP, DBP, and MBP, systolic, diastolic, and mean blood pressures, respectively; $\mathrm{PEP} / \mathrm{VET}$, ratio of preejection period to left ventricular ejection time; $\dot{\mathrm{V}}_{2}$, oxygen consumption; $\dot{\mathrm{V}}_{\mathrm{CO}}$, carbon dioxide production; $\dot{\mathrm{V}}_{\mathrm{E}}$, pulmonary ventilation; NS, not significant. 


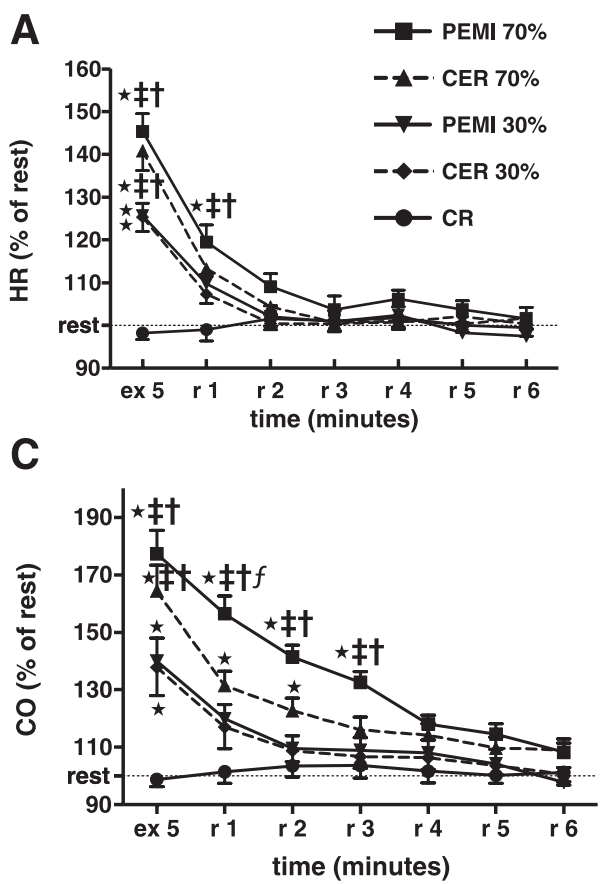

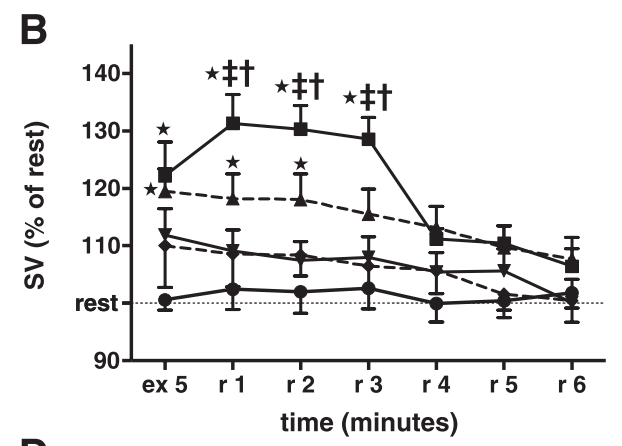

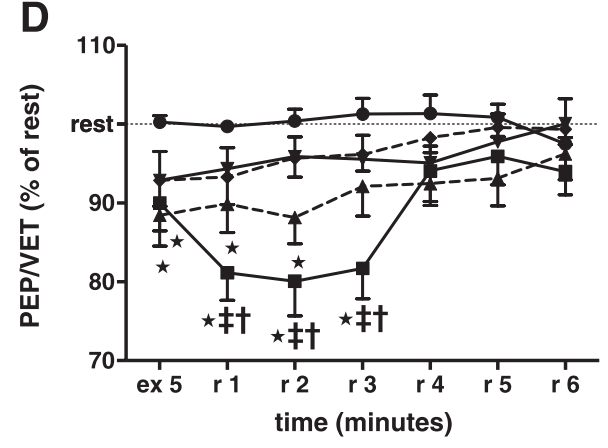

Fig. 1. Time courses of heart rate (HR, A), stroke volume (SV, B), cardiac output (CO, $C$ ), and inverse of myocardial contractility [preejection period-to-left ventricular ejection time ratio (PEP/VET), D] at the 5 th $\mathrm{min}$ of exercise (ex 5) and during 6 min of recovery (r1-r6) in all protocol sessions. Horizontal dashed line identifies resting level of variables. Values are means \pm SE percentages of rest. $\star P<0.05$ vs. control-rest (CR) test; $\ddagger P<$ 0.05 vs. postexercise muscle ischemia (PEMI) $30 \%$ test $\dagger P<0.05$ vs. control exercise recovery (CER) $30 \%$ test; ${ }^{f} P<0.05$ vs. CER $70 \%$ test. test, this parameter gradually decreased, reaching the CR level at the $3 \mathrm{rd}$ min of recovery. On the contrary, contractility further augmented (i.e., PEP/VET ratio decreased) throughout the circulatory occlusion time in the PEMI $70 \%$ test. During this period, contractility of PEMI 70\% was statistically higher than contractility in the PEMI 30\% and CER 30\% tests. As was described for SV, the high contractility observed during the PEMI 70\% test promptly decreased (i.e., PEP/VET ratio increased) once the thigh cuff was deflated.

During the 5th min of exercise, SBP (Fig. 2A) increased to a similar extent during all effort sessions compared with that in the CR test. SBP returned to the CR level already at the 1 st min of recovery in both the CER $70 \%$ and CER $30 \%$ tests. In contrast, during the entire period of circulatory occlusion in both the PEMI 70\% and PEMI 30\% tests, SBP resulted higher than during the CR test. Furthermore, at the 2nd min of recovery in the PEMI 30\% test, SBP resulted higher than the value observed during the CER $70 \%$ and CER $30 \%$ tests. Moreover, during the 2nd and 3rd min of occlusion, SBP was higher during the PEMI $70 \%$ test than during the CER $70 \%$ test. SBP in the PEMI $70 \%$ was also higher than in the CER $30 \%$ test from the 1 st to the 3 rd min of recovery.
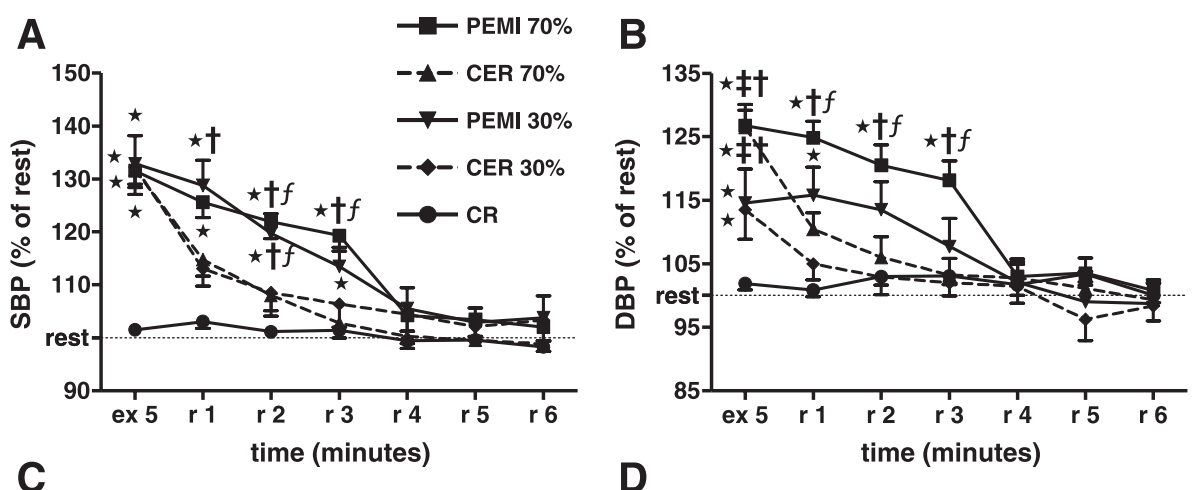

Fig. 2. Time courses of systolic blood pressure (SBP, $A$ ), diastolic blood pressure (DBP, $B$ ), mean blood pressure (MBP, $C$ ), and systemic vascular resistance (SVR, $D)$ at the 5 th min of exercise and during 6 min of recovery in all protocol sessions. Horizontal dashed line identifies resting level of variables. Values are means \pm SE percentages of rest. $\star P<0.05$ vs. CR test; $\ddagger P<0.05$ vs. PEMI

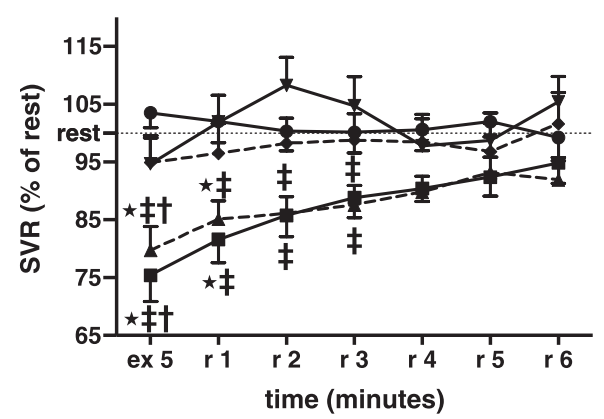
$30 \%$ test; $\dagger P<0.05$ vs. CER 30\% test; ${ }^{f} P<0.05$ vs. CER $70 \%$ test. 
Diastolic blood pressure (Fig. 2B) increased in response to exercise sessions at both intensities with respect to the CR test. However, this increment was more pronounced during exercises performed at $70 \%$ than at $30 \%$ of $\mathrm{W}_{\text {max }}$. During recoveries of both CER tests (i.e., without circulatory occlusion), DBP then swiftly returned to the CR level, which was reached already at the 1st min. Recovery sessions with circulatory occlusion (PEMI) were characterized by a DBP higher than in the CR test, and this fact was particularly evident in the PEMI $70 \%$ test when DBP was higher than CR during the entire period of cuff inflation and also higher than during the CER tests. In the postocclusion period (r4-r6), DBP was similar in all tests.

Figure $2 C$ depicts MBP time course, which increased during the 5th min of effort in all tests. As for SBP and DBP, during both CER tests, mean blood pressure promptly returned to CR values once the exercise ceased. On the contrary, MBP maintained high values in response to leg circulatory occlusion during both PEMI 70\% and PEMI 30\% tests. In particular, during the PEMI 70\% test, MBP was higher than in CER 70\%, CER 30\%, and CR for the entire period of cuff inflation. In the postocclusion period (r4-r6) MBP was similar in all tests.

SVR (Fig. 2D) decreased during the exercise periods in both PEMI $70 \%$ and CER 70\% tests but not during PEMI 30\% and CER 30\% tests. During recovery, SVR in both PEMI 70\% and CER 70\% tests resulted lower than during the CR test until the $1^{\text {st }}$ min of recovery only, when SVR promptly returned to the $\mathrm{CR}$ level. It is noteworthy that during the period of circulatory occlusion in the PEMI $30 \%$ test, SVR tended to increase, thus resulting higher than during both the PEMI 70\% and CER 70\% tests. This tendency of SVR to increase during PEMI 30\% was not present during CER 30\%.

Finally, Fig. 3, $A-D$, depicts time courses of ventilatory data. In detail, $\dot{\mathrm{V}}_{\mathrm{E}}$ (Fig. $3 A$ ) rose more during PEMI $70 \%$ and CER
70\% compared with that in PEMI 30\% and CER 30\% tests. This parameter returned to the CR level at the 1st min of recovery of PEMI 30\% and CER 30\% and at the 3rd min of recovery of PEMI $70 \%$ and CER 70\%. Similar behavior was shown by $\dot{\mathrm{V}}_{\mathrm{CO}_{2}}$ (Fig. $3 \mathrm{C}$ ), whereas $\dot{\mathrm{V}}_{2}$ returned to the $\mathrm{CR}$ level more rapidly than the other respiratory variables (Fig. $3 B$ ). It is noteworthy that there were no differences between the PEMI and CER tests: differences during the exercise and recovery period were mainly dependent on the entities of efforts. As concerns $\mathrm{CO}_{\text {2excess }}$ (Fig. $3 D$ ), this parameter increased during the exercise periods of PEMI $70 \%$ and CER $70 \%$ tests with respect to CR, PEMI 30\%, and CER 30\% tests. This increment lasted until the 3rd min of recovery, then $\mathrm{CO}_{2 \text { excess }}$ gradually returned to $\mathrm{CR}$ level. In contrast, PEMI $30 \%$ and CER 30\% tests did not induce any elevation in this variable compared with $\mathrm{CR}$ test.

\section{DISCUSSION}

The present study conducted on healthy humans provides two main findings. First, the muscle metaboreflex is capable of modulating heart contractility. Second, the intensity of the effort that precedes the muscle ischemia maneuver affects the cardiovascular responses to metaboreflex engagement. Indeed, the exercise conducted against the heavier workload $(70 \%$ of $\mathrm{W}_{\max }$ ) led to different responses during the period of PEMI in terms of blood pressure, peripheral, and central hemodynamic conditions with respect to the milder workload ( $30 \%$ of $\left.\mathrm{W}_{\max }\right)$. It is intriguing that after the milder workload, the metaboreflex relied mainly on vascular resistance increase, whereas after the heavier workload, the reflex relied mainly, and surprisingly, on the increase in myocardial contractility and SV.

The data from this study confirm that metaboreflex, elicited by circulatory arrest in the exercised muscle at the end of
Fig. 3. Time courses of pulmonary ventilation $\left(\mathrm{V}_{\mathrm{E}}, A\right)$, oxygen consumption $\left(\mathrm{V}_{\mathrm{O}_{2}}, B\right)$, carbon dioxide production $\left(\mathrm{V}_{\mathrm{CO}_{2}}, \mathrm{C}\right)$, and excess of carbon dioxide production $\left(\mathrm{CO}_{\text {2excess }}, D\right)$ at the 5 th min of exercise and during $6 \mathrm{~min}$ of recovery in all protocol sessions. Horizontal dashed line identifies resting level of variables. Values are means \pm SE percentages of rest. $\star P<0.05$ vs. CR test; $\ddagger P<0.05$ vs. PEMI $30 \%$ test; $\dagger P<0.05$ vs. CER $30 \%$ test.
A

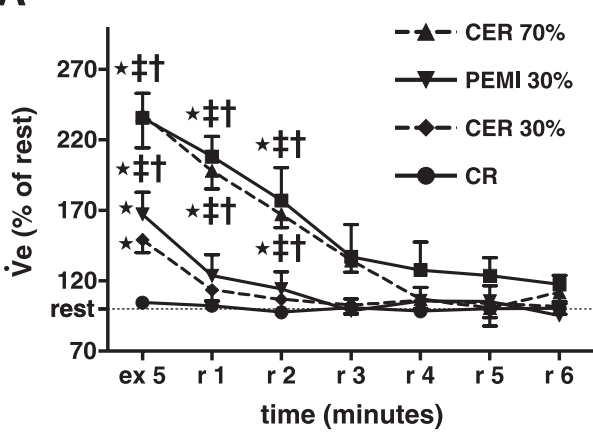

C

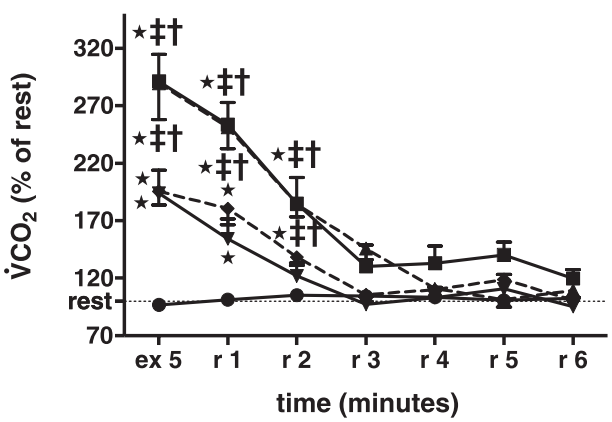

B

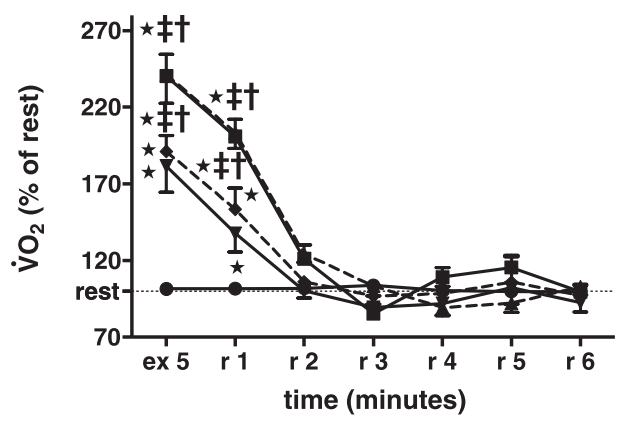

D

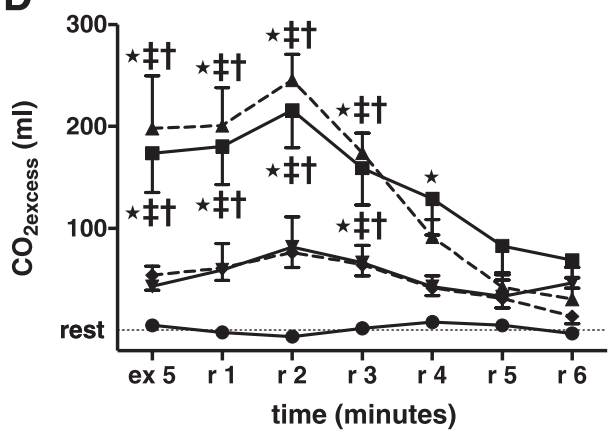


strain, induces a blood pressure response in humans (1a, 9, 29). It appears that work intensity affected the degree of blood pressure increment during PEMI, because exercise performed at $70 \%$ of $\mathrm{W}_{\max }$ caused a more pronounced MBP rise than the strain conducted against $30 \%$ of $\mathrm{W}_{\max }$. This result is in agreement with previous findings showing that work intensity exerted a strong effect on the magnitude of blood pressure response during muscle ischemia (7), and this phenomenon was explained by the fact that the degree of metaboreflex engagement is proportional to metabolite accumulation and $\mathrm{pH}$ fall within muscle $(7,22,32)$. It is likely that, in our setting, the exercise performed at the heavier workload caused more metabolite production, as testified by $\mathrm{CO}_{2 \text { excess }}$ behavior, and end-product accumulation and muscle acidification, which, in turn, evoked a more powerful metaboreflex recruitment.

As concerns the mechanisms underlying this blood pressure behavior, it is noteworthy that the workload before PEMI appeared to markedly affect both the central and peripheral hemodynamic response. In particular, the blood pressure response was mainly achieved via a flow increment (i.e., $\mathrm{CO}$ increase) during PEMI after the $70 \% \mathrm{~W}_{\max }$ test, whereas after the $30 \%$ test it was ascribable predominantly to vasoconstriction (i.e., SVR increase). The increase in CO during PEMI after $70 \%$ of $\mathrm{W}_{\max }$ was mostly due to the evident rise in SV. In fact, during PEMI 70\%, HR was higher than the other protocol sessions during the 1st min of cuff inflation only. This result is in agreement with the concept that metaboreflex recruitment during postexercise ischemia has little or no influence on HR (1a, 9, 25, 29). To explain this HR feature, it has been proposed that the metaboreflex-induced increase in sympathetic tone, which occurs during PEMI, may be counterbalanced by the concomitant baroreflex-induced rise in parasympathetic activity along with the withdrawal of central command $(18,23,25)$.

The SV increment during the PEMI $70 \%$ test was the consequence of the enhancement in contractility that occurred during the whole period of circulatory occlusion, as testified by the PEP/VET ratio decrease. In contrast, during the PEMI 30\% test, this contractility behavior was not present; actually, in this test the blood pressure increment was totally due to the increase in SVR, which showed values higher than in the PEMI $70 \%$ and CER 70\% tests for the entire duration of circulatory occlusion. This SVR response promptly ceased once the cuff was deflated so that SVR returned to a level no different from that of the other protocol conditions. In other words, after the strain performed at the lighter level $\left(30 \%\right.$ of $\left.\mathrm{W}_{\max }\right)$, blood pressure remained at higher values with respect to baseline via an increase in SVR with little, if any, contribution of CO recovery. These results were at variance with previous works conducted in animal models, which reported that the cardiovascular response to metaboreflex relied mainly on CO during mild exercise, whereas peripheral vasoconstriction became more important as the intensity of exercise rose $(1,35,41)$. These results also appear to be at odds with a recent report from Piepoli's laboratory (9) where it was concluded that the blood pressure response during metaboreflex activation after mild rhythmic handgrip performed at $30 \%$ of $\mathrm{W}_{\max }$ depends on the capacity to increase CO rather than SVR. The consensus was that if the cardiac reserve could still be used (i.e., if the contractility was not fully used during exercise), then the metaboreflex-induced pressure response relied mainly on CO. In contrast, if there is no cardiac reserve further available for increasing $\mathrm{SV}$, as there is during and/or after strenuous efforts, then the response relies on peripheral vasoconstriction and SVR increment. Therefore, our results are opposite from what was expected from animal studies $(1,35,41)$ and from a previous report from Piepoli's laboratory (9).

An explanation of the contractility and SV responses observed in our study may derive from the aforementioned interaction that exists between metaboreflex and baroreflex. It has recently been proposed (19) that the latter reflex counteracts a metaboreflex-induced increase in blood pressure via a greater buffering of sympathetic activity directed to vascular tone regulation, and yet, metaboreflex exerts greater control over sympathetic activity to the heart. In our experiment, baroreflex was probably stimulated more during the PEMI 70\% than during the PEMI 30\% test, because the mean arterial pressure (MAP) level was higher during the first condition. Thus, during the PEMI $70 \%$ setting, there was probably a pronounced baroreflex-mediated vasodilatation that opposed the increased sympathetic discharge due to metaboreflex engagement. Moreover, it has been reported that an increase in blood pressure may stimulate intracardiac receptors that specifically mediate vasodilatation (17). In fact, the PEMI 70\% test induced no rise in SVR, as is to be expected when a sympathetic overstimulation takes place. It is thus likely that baroreflex and/or cardioreflex successfully counterbalanced the metaboreflex-sympathetic discharge toward the vessels during PEMI 70\%, whereas the metaboreflex-mediated sympathetic stimulation of the heart may operate, resulting in an enhanced contractility and SV increase. However, this occurrence must presuppose the existence of a contractility reserve. Indeed, it is likely that the contractility reserve was not totally used during efforts. In fact, as depicted in Fig. $1 B$, the SV increment at the 5th min of exercise of the two tests performed against $70 \%$ of $\mathrm{W}_{\max }$ was on average $\sim 20 \%$ above the resting level. These data indicate that the magnitude of SV engagement during exercises performed at this intensity was not at its maximum, because healthy young people, such as those enrolled in the present study, can increase SV up to $50-60 \%$ of the resting values in response to heavy exercise $(11,30)$. Thus it is likely that a single-leg knee extension is not enough to fully recruit the contractility reserve of healthy young subjects. As a consequence, during both PEMI 70\% sessions, there was still the possibility to further increase myocardial contractility and, in turn, SV to achieve the blood pressure increment.

However, despite the fact that SV augmentation was even less evident during the efforts against $30 \%$ of $\mathrm{W}_{\max }(+10 \%$ only), this mechanism of a further increase in contractility was not utilized during the PEMI $30 \%$ test. A possible explanation for the lack of a contractility response during the PEMI $30 \%$ test may be the existence of a threshold for contractility recruitment; i.e., the contractility reserve is recruited only when the degree of sympathetic discharge due to metaboreflex stimulation reaches a threshold. In this light, an enhancement in SV is possible only when this threshold is passed. Indeed, the existence of a threshold during metaboreflex recruitment has already been demonstrated for blood pressure and has been related to muscle $\mathrm{pH}$. Furthermore, it has been found that once this threshold is passed, MAP increases linearly with the decrease in muscular $\mathrm{pH}$ (22). Thus, in our experiment, it may be that there was a more pronounced muscle lactate and $\mathrm{H}^{+}$ accumulation during strains performed at $70 \%$ of $\mathrm{W}_{\max }$ than 
during those against $30 \%$ of $\mathrm{W}_{\max }$, as demonstrated by the

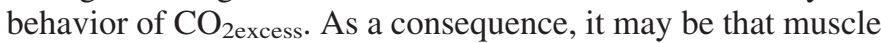
$\mathrm{pH}$ dropped more and that the threshold for contractility recruitment was passed only after the heavier strain. Hence, it is possible to speculate that metaboreflex was stimulated more after the exercise performed at $70 \%$ of $\mathrm{W}_{\max }$, and this fact may have caused the different contractility and SV pattern. It may be argued that metaboreceptors that are stimulated by a small $\mathrm{pH}$ decrease may activate sympathetic fibers direct to the vessels, thus inducing vasoconstriction. A lower $\mathrm{pH}$ may activate receptors more intensely, or different receptors, triggering the activation of sympathetic fibers direct to the heart. However, when the latter condition occurs, baroreflex vasodilatation also occurs. The latter effect prevails over sympathetic activation, and SVR does not increase.

As far as the different findings from a previous report (9) are concerned, it should be considered that the exercise protocol employed in that work (rhythmic handgrip) involved the arm musculature, which is known to be composed of more fasttwitch glycolitic fibers than the legs. For a given level of force production, the fast-twitch fibers produce a greater amount of end products than slow-twitch fibers (15). It may be thus argued that in that setting (arm exercise at $30 \%$ of $\mathrm{W}_{\max }$ ) there was a more pronounced metabolite accumulation during the mild exercise than in the present report (leg exercise at $30 \%$ of $\left.\mathrm{W}_{\max }\right)$, and this may have produced a more intense metaboreflex stimulation, even though the relative level of force production was the same. Thus the different hemodynamic response between the two mild exercises employed in the two reports may be explained through the different type of musculature involved.

Whatever the cause, the results of the present study clearly indicate that contractility regulation is possible in human beings during the evoking of metaboreflex by the PEMI method and that this modulation depends on the intensity of the previous effort. To the best of our knowledge, this is the first study that analyzes such a hemodynamic feature during metaboreflex evoked by different workload intensities in the same individuals. However, it is to be noted that, surprisingly, there are very few works dealing with central hemodynamics during metaboreflex in humans (1a, 5, 6, 9), and further research is warranted to better characterize the phenomenon.

With respect to respiratory parameters, neither the PEMI $70 \%$ nor the PEMI $30 \%$ tests induced any change in $\dot{\mathrm{V}}_{\mathrm{E}}, \dot{\mathrm{V}}_{2}$, and $\dot{\mathrm{V}}_{2}$ compared with the corresponding CER session. The absence of any modification in these parameters during PEMI maneuvers suggests that the hemodynamic response was unaffected by any change in ventilatory pattern.

The data from the present work confirm previous findings reporting that the pressure response to metaboreflex stimulation by PEMI is not merely composed of reflex vasoconstriction, but it rather appears that hemodynamic regulation is a very complex and flexible phenomenon during which an interplay between myocardial contractility, HR, and peripheral vasoconstriction takes place because of the contemporary activation of both metaboreflex and baroreflex $(9,25,27)$. Hence, the blood pressure response is the result of a balance between flow-mediated and vasoconstriction-mediated mechanisms that operate on the basis of contractility reserve and of peripheral vasal tone regulation.
Limitations of this work. One possible limitation of the present work is that it did not assess the concentration of muscle metabolites to verify whether or not the heavier workload induced greater increase than the milder workload in those end products that are putatively responsible for metaboreceptors stimulation. Among these products there are $\mathrm{H}^{+}$, lactate, diprotonated phosphate, ATP, adenosine, arachidonic acid, prostaglandins, potassium, bradykinin, sodium, acetylcholine, histamine, serotonin, and others. To the best of our knowledge, there is conclusive evidence for none of these substances regarding its importance or even its real involvement in the metaboreflex. Because the dosage of such a great number of products is not very easy in humans and needs the utilization of complex and sometimes invasive procedures, such as muscle biopsy, we employed the $\mathrm{CO}_{2 \text { excess }}$ to obtain an index of lactate and $\mathrm{H}^{+}$production, which, by means of muscle acidification, are supposed to be major substances capable of evoking the metaboreflex $(32,36,37)$.

Another potential limitation is that we did not measure blood flow from a nonexercising limb to directly verify whether or not there were changes in the peripheral vascular resistance during the metaboreflex maneuvers. However, our results strongly suggest that a vasodilatation took place during the PEMI $70 \%$ test. In fact, in this condition (i.e., an occlusion of one leg circulation), an increase in SVR is to be expected because the mechanical hindrance of skeletal muscle blood flow is known to augment total peripheral resistance per se (38). However, we did not detect any difference between PEMI $70 \%$ and CER $70 \%$ tests in terms of SVR, and this fact leads us to speculate that a peripheral vasodilatation in the nonoccluded circulation occurred in the PEMI 70\% setting. The same reasoning, however, with opposite results can be applied to the strains conducted at milder workloads. In this setting, a clear tendency of SVR to increase was detected for PEMI 30\% test, and this fact indicates that a vasoconstriction was probably present during this period.

In conclusion, the present investigation provides evidence that hemodynamic regulation during metaboreflex engagement caused by postexercise muscle ischemia in humans is dependent on the intensity of the previous effort. It appears that in the presence of an available cardiac reserve and an adequate metaboreceptor stimulation, contractility modulation is possible. When both conditions occur, pressure response regulation shifts from peripheral vasoconstriction to a flow-increased mechanism. In the light of these data, the cardiovascular response during metaboreflex is not merely achieved by a systemic increase in vascular resistance, but it appears that there is a complex interplay between peripheral vascular regulation and heart contractility modulation.

\section{ACKNOWLEDGMENTS}

This study was supported by the University of Cagliari, the University of Torino, the University of Sassari, the Italian Ministry of the Scientific Research, and PRISMA Onlus.

\section{REFERENCES}

1. Augustyniak RA, Collins HL, Ansorge EJ, Rossi NF, and O'Leary DS. Severe exercise alters the strength and mechanisms of the muscle metaboreflex. Am J Physiol Heart Circ Physiol 280: H1645-H1652, 2001.

1a.Bastos BG, Williamson JW, Harrelson T, and Nôbrega AC. Left ventricular volumes and hemodynamic responses to postexercise ischemia in healthy humans. Med Sci Sports Exerc 32: 1114-1118, 2000. 
2. Beaver WL, Wasserman K, and Whipp BJ. Bicarbonate buffering of lactic acid generated during exercise. J Appl Physiol 60: 472-478, 1986

3. Belardinelli R, Ciampani N, Costantini C, Blandini A, and Purcaro A. Comparison of impedance cardiography with thermodilution and direct Fick methods for noninvasive measurement of stroke volume and cardiac output during incremental exercise in patients with ischemic cardiomyopathy. Am J Cardiol 77: 1293-1301, 1996.

4. Bernstein DP. A new stroke volume equation for thoracic electrical bioimpedance: theory and rationale. Crit Care Med 14: 904-909, 1986.

5. Bonde-Petersen F, Rowell LB, Murray RG, Blomqvist GG, White R, Karlsson E, Campbell W, and Mitchell JH. Role of cardiac output in the pressor responses to graded muscle ischemia in man. $J$ Appl Physiol 45: 574-580, 1978.

6. Bonde-Petersen F and Suzuki Y. Heart contractility at pressure loads induced by ischemia of exercised muscle in humans. $J$ Appl Physiol 52: 340-345, 1982.

7. Cornett JA, Herr MD, Gray KS, Smith MB, Yang QX, and Sinoway LI. Ischemic exercise and the muscle metaboreflex. J Appl Physiol 89: 1432-1436, 2000.

8. Crisafulli A, Orrù V, Melis F, Tocco F, and Concu A. Hemodynamics during active and passive recovery from a single bout of supramaximal exercise. Eur J Appl Physiol 89: 209-216, 2003.

9. Crisafulli A, Scott AC, Wensel R, Davos CH, Francis DP, Pagliaro P, Coats AJS, Concu A, and Piepoli MF. Muscle metaboreflex-induced increases in stroke volume. Med Sci Sports Exerc 35: 221-228, 2003.

10. Crisafulli A, Melis F, Tocco F, Santoboni UM, Lai C, Angioy G, Lorrai L, Pittau G, Concu A, and Pagliaro P. Exercise-induced and nitroglycerin-induced myocardial preconditioning improves hemodynamics in patients with angina. Am J Physiol Heart Circ Physiol 287: H235-H242, 2004.

11. Crisafulli A, Tocco F, Pittau G, Caria M, Lorrai L, Melis F, and Concu A. Detection of lactate threshold by including haemodynamic and oxygen extraction data. Physiol Meas 27: 85-97, 2006.

12. Crisafulli A, Pittau GL, Lorrai L, Cominu M, Tocco F, Melis F, and Concu A. Poor reliability of heart rate monitoring to assess oxygen consumption during field training. Int J Sports Med 27: 55-59, 2006.

13. Crisafulli A and Concu A. Exercise-induced cardiovascular adjustments by muscle receptors stimulation. In: Focus on Exercise and Health Research. Hauppauge, NY: Nova Science, 2006, p. 181-201.

14. Fadel PJ. Muscle metaboreflex-induced increases in stroke volume. Med Sci Sports Exerc (Commentary) 35: 229, 2003.

15. Fisher JP and White MJ. Muscle afferent contributions to the cardiovascular response to isometric exercise. Exp Physiol 89: 639-646, 2004.

17. Hainsworth R. Reflexes from the heart. Physiol Rev 71: 617-658, 1991.

18. Iellamo F, Pizzinelli P, Massaro M, Raimondi G, Peruzzi G, and Legramante JM. Muscle metaboreflex contribution to sinus node regulation during static exercise. Circulation 100: 27-32, 1999.

19. Kim JK, Sala-Mercado JA, Rodriguez J, Scislo TJ, and O'Leary DS. The arterial baroreflex alters the strength and mechanisms of the muscle metaboreflex pressor response during dynamic exercise. Am J Physiol Heart Circ Physiol 288: H1374-H1380, 2005.

20. Moran D, Epstein Y, Keren G, Laor A, Sherez J, and Shapiro Y. Calculation of mean arterial pressure during exercise as a function of heart rate. Appl Human Sci 14: 293-295, 1995.

21. Nelsen RA and Dimsdale JE. Use of radial arterial tonometric continuous blood pressure measurement in cardiovascular reactivity studies. Blood Press Monit 7: 259-263, 2002.

22. Nishiyasu T, Ueno H, Nishiyasu M, Tan N, Morimoto K, Morimoto A, Deguchi T, and Murakami N. Relationship between mean arterial pressure and muscle $\mathrm{pH}$ during forearm ischemia after sustained handgrip. Acta Physiol Scand 151: 143-148, 1994.
23. Nishiyasu T, Nobusuke T, Morimoto K, Nishiyasu M, Yamaguchi Y, and Murakami N. Enhancement of parasympathetic cardiac activity during activation of muscle metaboreflex in humans. J Appl Physiol 77: 2778-2783, 1994.

24. Northridge DB, Findlay IN, Wilson J, Henderson E, and Dargie HJ. Non-invasive determination of cardiac output by Doppler echocardiography and electrical bioimpedance. Br Heart J 63: 63-93, 1990.

25. O'Leary DS. Autonomic mechanisms of muscle metaboreflex control of heart rate. J Appl Physiol 74: 1748-1754, 1993.

26. O'Leary DS and Sheriff DD. Is the muscle metaboreflex important in control of blood flow to ischemic active skeletal muscle in dogs? Am J Physiol Heart Circ Physiol 268: H980-H986, 1995.

27. O'Leary DS and Augustyniak RA. Muscle metaboreflex increases ventricular performance in conscious dogs. Am J Physiol Heart Circ Physiol 275: H220-H224, 1998.

28. O'Leary DS, Augustyniak RA, Ansorge EJ, and Collins H. Muscle metaboreflex improves $\mathrm{O}_{2}$ delivery to ischemic active skeletal muscle. Am J Physiol Heart Circ Physiol 276: H1399-H1403, 1999.

29. Piepoli M, Clark AL, and Coats AJS. Muscle metaboreceptors in hemodynamic, autonomic, and ventilatory responses to exercise in men. Am J Physiol Heart Circ Physiol 269: H1428-H1436, 1995.

30. Plotnick GD, Becker LC, Fisher ML, Gerstenblith G, Renlund DG, Fleg JL, Weisfeldt ML, and Lakatta EG. Use of the Frank-Starling mechanism during submaximal versus maximal upright exercise. Am J Physiol Heart Circ Physiol 251: H1101-H1105, 1986.

31. Roeker K, Mayer F, Striegel H, and Dixkhuth HH. Increase characteristics of the cumulated excess- $\mathrm{CO}_{2}$ and the lactate concentration during exercise. Int J Sports Med 21: 419-423, 2000.

32. Rowell LB and O'Leary DS. Reflex control of the circulation during exercise: chemoreflexes and mechanoreflexes. J Appl Physiol 69: 407418, 1990.

33. Sala-Mercado JA, Hammond RL, Kim JK, Rossi NF, Stephenson LW, and O'Leary DS. Muscle metaboreflex control of ventricular contractility during dynamic exercise. Am J Physiol Heart Circ Physiol 290: H751H757, 2006.

34. Sheriff DD, Wyss C, Rowell L, and Scher A. Does inadequate oxygen delivery trigger pressor response to muscle hypoperfusion during exercise? Am J Physiol Heart Circ Physiol 253: H1199-H1207, 1987.

35. Sheriff DD, Augstyniak RA, and O'Leary DS. Muscle chemoreflexinduced increases in right atrial pressure. Am J Physiol Heart Circ Physiol 275: H767-H775, 1998.

36. Shoemaker JK, Kunselman AR, Silber DH, and Sinoway LI. Maintained exercise pressor reflex in heart failure. J Appl Physiol 85: 17931799, 1998.

37. Sinoway $\mathbf{L I}$ and $\mathbf{L i} \mathbf{J}$. A perspective on the muscle reflex: implications for congestive heart failure. J Appl Physiol 99: 5-22, 2005.

38. Stebbins CL, Brown B, Levin D, and Longhurst JC. Reflex effect of skeletal muscle mechanoreceptor stimulation on the cardiovascular system. J Appl Physiol 65: 1539-1547, 1988.

39. Strange S, Secher NH, Pawelczyk JA, Karpakka J, Christensen NJ, Mitchell JH, and Saltin B. Neural control of cardiovascular responses and of ventilation during dynamic exercise in man. $J$ Physiol 470: 693-704, 1993.

40. Thornton JM, Aziz T, Sclungam D, and Paterson DJ. Electrical stimulation of the midbrain increases heart rate and arterial blood pressure in awake humans. J Physiol 539: 615-621, 2002.

41. Wyss C, Ardell JL, Scher AM, and Rowell LB. Cardiovascular responses to graded reductions in hindlimb perfusion in exercising dogs. Am J Physiol Heart Circ Physiol 245: H481-H486, 1983. 